\title{
Providing Language Interfaces with Robotic Process Automation and Text Retrieval for automated integration of applications and unstructured data
}

\author{
[Research-in-Progress]
}

Andreas Niekler, Leipzig University, Augustusplatz 10, 04103 Leipzig, Germany aniekler@informatik.uni-leipzig.de

Mark Busse, $1000^{\circ}$ Digital GmbH, Mozartstraße 3, 04107 Leipzig, Germany mark.busse@1000grad.de

Matthias Gulde, Leipzig University, Augustusplatz 10, 04103 Leipzig, Germany mt.delgu@gmail.com

Lino Markfort, Leipzig University, Augustusplatz 10, 04103 Leipzig, Germany lino.markfort@uni-leipzig.de

Felix Helfer, Leipzig University, Augustusplatz 10, 04103 Leipzig, Germany helfer@informatik.uni-leipzig.de

\begin{abstract}
In this paper we show the outline of an integration of unstructured data and Robotic Process Automation (RPA) approaches into a chatbot framework. We describe how RPA applications are connected to a chatbot system and show a possible system sketch. Furthermore, we describe the integration of Open Question Answering techniques like DocChat, semantic clustering and the Universal Sentence Encoder in order to acquire direct answers to user questions from documents. From this, we derive a standalone bot framework that we will use in the future for deployment in industrial contexts. For this purpose, we integrate the tools in a user and application-oriented way in the near future.
\end{abstract}

Keywords: Chatbot, Conversational Interface, Natural Language Processing, Information Retrieval, Robotic Process Automation

\section{Introduction}

Chatbots promise industry-related application areas for communication in fields such as customer service, compliance management or human resources (Adam et al., 2020; Feine et al., 2019; Følstad \& Skjuve, 2019; Jain et al., 2018). In customer service, applications for support requests or customer information have become an established field. Above all, alternative access to Frequently Asked Questions (FAQ) sections or similar information is in demand and better accepted by users. In the area of human resources, relatively standardized tasks such as application processes, employee communication, compliance information or standardized information can be passed on through these systems in the first step before a personal contact with the employees. In particular, this simplification allows staff to handle difficult requests with high while simple information can be easily passed on automatically. Beyond industrial applications, this technology can also be used by cities and municipalities to ease contact with citizens and access to municipal 
services. For example, citizens can use this interface to get quick access to information and services such as applying for ID documents, registration, property management or waste disposal.

With regard to the functionality and maintenance of chatbots, a number of frameworks and industrial solutions are now available. Most solutions focus on the functionality of modeling conversations and conversation flows. We know the concept behind this as Intent detection. An Intent is a specific information interest of the user, which has to be recognized automatically by a text input to the system. The technological basis for this is Natural Language Understanding. This discipline refers to technologies that enable the understanding of natural language by software. In most modern applications, this is realized via pattern matching, machine learning and deep learning technologies. The user's speech input is read by a classification component, usually based on deep learning, and assigned to an Intent stored in the system. If this is unsuccessful, the system can evade or request further information accordingly. Intents in the form of Smalltalk components are stored for basic communication or for more trivial conversations, so that a conversation can be conducted naturally and irrelevant inputs can still be answered with natural responses (i.e. "How are you", “Whats's up", "Yes I'm a robot but I'm a smart one!", “Are you real?”). This is especially intended to create the feeling of a natural language conversation and serves the acceptance by the users. The actual Intents, i.e., the information to be offered in the chatbot application, must be stored in a configuration environment. Many frameworks offer wizards or Content Management Systems (CMS) for this purpose. One exchange format are table documents that map the complete Intent structure via spreadsheets. Intents are defined by keywords or concretely formulated questions and variants thereof, so that a system can be trained with them. The training goal of the Machine Learning backends in those systems is an association between a text input and a possible answer or conversation thread in the system. The strong relationship to Question Answering tasks is indisputable, but here they are embedded in a conversation. Normally, the number of Intents exceeds several thousand conversation threads and pieces of information for applications in the industrial context, especially for the applications mentioned above. This requires separate content managers and processes in the companies and makes the maintenance and initial configuration of chatbots very time-consuming and represents a high entry threshold for companies. This is especially problematic because the benefits of conversational interfaces, e.g. chatbots, are still highly debated (Brandtzaeg \& Følstad, 2017; Dale, 2016; Shawar \& Atwell, 2007). On the contrary, most of the information that is to be offered via Conversational Interfaces is already stored in other specialized applications, CMSs, or documentations. Users usually have the impression that duplicate structures must be maintained here, since information integration is not yet guaranteed via standard interfaces.

To solve these problems, interfaces to external applications can be used and queries are translated into a compatible query. For example, we already use a simple integration about the garbage emptying of a municipality. A homeowner is unsure whether and which trash can needs to be provided for disposal. The garbage can is full or overflowing and further waste is present. Again and again the same question arises: "When will the garbage can be collected?" The existing online schedule on the company's website is not yet sufficient. For this purpose, the electronic calendar of the waste disposal company can be connected to a chatbot system. With an Intent, we detect if users ask about garbage emptying and extract already meaningful information such as the type of garbage. For example, users can ask "When will the household garbage be disposed". The bot registers this intention and can ask back "For what street?". With an appropriate answer, a street 
name can be detected in the text and the combination of the type of garbage and the street name results in a query that we can submit to the service ("Next week, Friday the household garbage will be emptied in the example street."). Frequent callers to the municipality are citizens who currently have no Internet access (because they are on the road, for example) or who prefer the telephone use to internet use. Connecting the chatbot to a phone channel is additionally possible via multiple existing commercial services. This also enables exclusive telephone forwarding to a service representative for further questions. For such recurring questions, this is a real economic benefit. Surely this is a very simple integration but an important step towards the integration of business applications. Therefore, in the next sections we will show an advanced integration and opening of chatbots through modern approaches of information integration. Unfortunately, not all business applications today have responsive interfaces other than a user interface, and much information is also stored in documents. In our contribution we therefore show how Robotic Process Automation (RPA) and modern approaches of Text Retrieval and Question Answering (QA) can be used to integrate information from documents and specialized applications directly into Conversational Interfaces. We present our application concept and display concrete examples from our work.

\section{RPA and external applications in Conversational Interfaces}

A user Intent can be associated with an external response web service instead of a static response. The web service is invoked with the user's detected entities and parameters. The return value of the call can be used in a dynamically generated response to the user. In this way, for example, a database query can be executed or an RPA request can be started. Sometimes, only an RPA sequence is launched for execution. In Figure 1, we show our system sketch and the integration of external RPA tasks. For example, form processes, ordering procedures, and information about additional services from external specialist applications can be connected to the Conversational Interface. We use the concept of subbots in our system. This extension helps us to encapsulate small tasks in submodules, so that we achieve a better structure and administrability of individual tasks. In the graphic, different subbots are shown here, performing different tasks. Internal subbots control different user intentions, such as conversations in different topic areas. External subbots respond to Intents that require for example business applications. Interfaces and transfer information can then be defined in this way. In our framework we implemented the aforementioned pilot study where we have connected the information for garbage collection in a municipality to the system. When the Intent to request collection times via the Conversational Interface is detected, the request is forwarded to an external subbot. This subbot requests the specific address or takes it from the Intent and translates this into a query for a database where this information is stored. With the response, the web service generates a return value and our chatbot system is able to formulate the answer.

RPA is an approach in which repetitive, manual, time-consuming or error-prone activities are learned and automated by so-called software robots. These not only use technical APIs of system components, but can also control application software via the presentation layer, comparable to execution by a human. RPA combines workflow modeling, scripting, and job scheduling. Accordingly, an RPA application serves our chatbot framework as an additional interface or adapter to individual customer specialized applications that do not have their own modern web 
service interface. Via RPA, a search query can be converted into simulated keyboard and mouse input. The result display on the screen can then be determined, e.g. via an integrated visual analysis tool, and transferred to the response service of a chatbot. Further possibilities are e.g. the translation of a user intention into database queries, form input or the general sequential execution of user interaction with a specific goal.

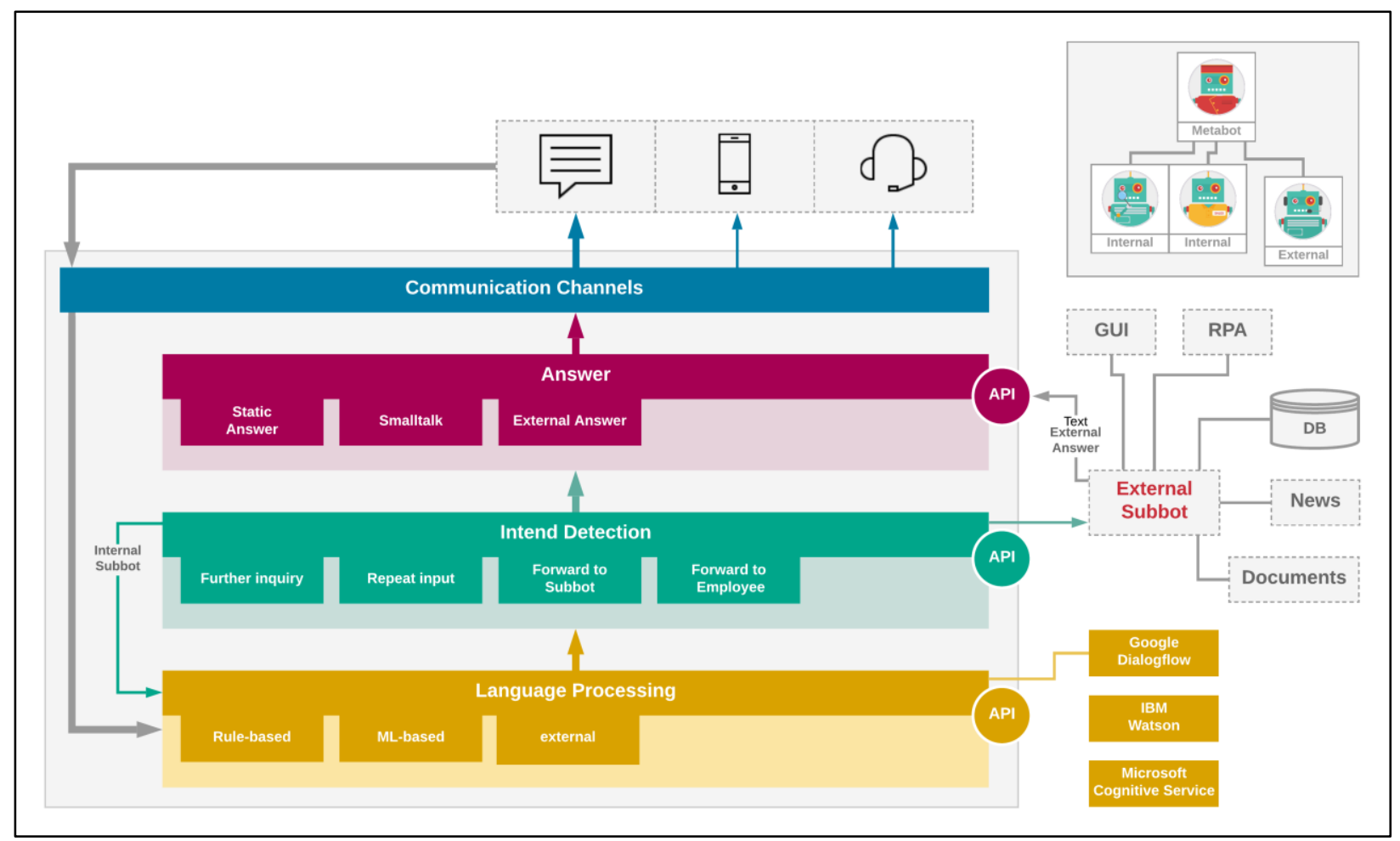

Figure 1: Chatbot System Architecture including RPA and Document Retrieval. Please perceive, we are also using external language processing capabilities like Google Dialogflow. This shows that those frameworks are enablers for more complex applications like our proposed system.

Here we present specific experiences with the integration of the specialist application Zoho-People (https://www.zoho.com/people/) to determine the remaining vacation days of an employee. In the scenario "Determine open vacation days", users $\log$ in the intranet (e.g. via Atlassian Confluence). A token with a user ID / unique user name is transferred internally during the chat integration. A virtual Windows workstation is started on a Microsoft server in the cloud. The RPA client of "Automation Anywhere" is started on the virtual workstation. In the next step, users request the number of vacation days left via the Chatbot, which recognizes this Intent and forwards it to the RPA web service interface. The RPA task to determine the users vacation days is then sent to the RPA task server in a queue. By executing the task from the queue, the system can then generate a response for the chat interface. This task is accomplished by simulating a browser interaction, after which mouse movements and clicks on specific areas are executed. On the screen, a visual analysis tool searches for a pattern (phrase "paid vacation" followed by a statement). This determined number is the answer which can be output in the chat system.

\section{Text Retrieval and Question Answering in Conversational Interfaces}


Through modern approaches of Text Retrieval and QA, it is possible to embed text documents and the concepts they contain directly into Conversational Interfaces. These technologies allow us to access search processes directly through natural language input or questions. For our application we integrate two solutions. On the one hand, we combine automatic keyword extraction with semantic clustering, so that content-coherent sections in documents can be grouped via an Intent and can be queried by users. Second, we integrate QA technologies that can directly map natural language input and questions to text areas with potential answers. This allows us to use the Conversational Interface to access information in text documents, and eliminates the need for the time-consuming transfer of this content into the Intent structure of Chatbots in the future.

In the case of users cannot formulate a specific question but have a particular interest in information, we divide documents into meaningful sections like Paragraphs or Chapters. We then extract significant keywords from the sections using a log-likelihood significance test (Dunning, 1993). As reference we use general linguistic formulations from 4 million German sentences (Quasthoff et al., 2015). Each section is then represented by a set of keywords. Subsequently, we assign FastText embeddings to each keyword so that they are semantically encoded (Bojanowski et al., 2016). This allows us to represent each paragraph by the mean of the semantic vectors. Since it is unwieldy to select a paragraph directly via the Conversational Interface using a selection of keywords, we now introduce a clustering process. In a first step, we assign each semantic representation of a paragraph to one of 4 clusters. We realize this with a k-means clustering. Each cluster is now described from the semantic paragraph representations that are most similar. From a taxonomy or list of possible head terms for a domain, we assign a proxy term to each cluster so that a cluster is represented using a single term. This leads to the following process for navigating a text document using a Conversational Interface: Users now select a proxy term via the voice interface, which leads to a reduction of the search set of text paragraphs assigned to this cluster. The remaining paragraphs are transferred back into 4 clusters with a generic term and a new, more specific selection by the user can be achieved. This is repeated until one paragraph remains in the search set. This paragraph then can be displayed or read aloud in the document context. The document and the information thus can be accessed without having to store each piece of information as an Intent and without users needing to know the specific terms for access. This can be very useful for documents with heterogeneous information, such as data protection or questions about parental leave, since users often search for information without having specific terms for the query at hand.

Our second approach tries to retrieve answers directly from a text basis. Automated QA describes the task "to retrieve small snippets of text that contain the actual answer to a question rather than the document lists traditionally returned by text retrieval systems" (Voorhees et al., 1999, S. 8), classifying it as a subfield or advanced form of information retrieval (IR), while other authors also describe it as a joint subfield of IR and artificial intelligence (Mollá \& Vicedo, 2007). Compared to traditional IR systems retrieving only the actual answer to a question is of advantage to the users saving a lot of time if they only search for a specific bit of information instead of more comprehensive sources about their topics of interest. The possibility to ask questions in natural language furthermore guarantees the intuitive use of QA systems and allows for more complex requests than purely keyword-based searches - making such systems a valuable tool for quickly extracting information from any large collection of unstructured or semi structured documents. More precisely, the type of application, which is the focus of this work, would be categorized as 
open-domain. Over the last years, the field of QA has seen rapid development, with QA system Watson famously winning against the two highest ranked players in the TV quiz show "Jeopardy!" in 2011 (Ferrucci et al., 2013) and the emergence of new machine-learning based technologies that greatly advanced the state of the art for QA, most notably BERT (Devlin et al., 2018). DocChat (Yan et al., 2016) is one such system, that is basically an ensemble of different answer selection techniques of varying complexity and requiring varying amounts of training data. DocChat is a QA system designed as a chatbot engine that operates on an arbitrary text corpus. Given any question, the desired behavior of the application is to return the sentence from the corpus which best fits as an answer, or to return nothing if the corpus does not contain an answer. On the top level, DocChat consists of three components that are invoked sequentially for each question: response retrieval, collecting answer candidates from the corpus, response ranking, reranking those using more complex (and computationally more expensive) methods, and lastly response triggering, deciding whether or not the highest ranked answer candidate is actually a valid answer (Yan et al., 2016). Response rankings are the most extensive of these components as they encompass multiple independent methods of estimating answer quality. Those can be divided into the categories word-, phrase- and sentence-level, that each only take the respective units of text into consideration, document-level, which includes the context of answer candidates, relationand type-level, focusing on the relation between subject and object of the sought statement and the type of the object, respectively. The last category is the topic-level, where the similarity between question and answer is calculated based on topic models. A machine-learned ranking model is then used to compute a single score from the values a question-answer pair scored in all the features (Yan et al., 2016). We also combined this approach with the Google Sentence Encoder in order to extend the retrieval performance with modern deep learning based self-attention language model capabilities (Cer et al., 2018). To demonstrate accessing answers from text documents a bit more vividly, we have shown a screen capture from our application demo in Figure 2. For this demo, we have prepared Wikipedia articles by physicists and these are used directly to answer questions. 

.
Hello, I'm the $1000^{\circ}$ Bot and I'm happy to answer your questions.
(\$) $\mathrm{Hi}$ !
You can ask the DocChat system to try to find an answer to question about sci- entists.

\section{What did Merkel study?}
(8) Merkel was educated at Karl Marx University, Leipzig, where she studied phy-
sics from 1973 to 1978.
Who invented relativity theory?
$\odot$
Einstein originally framed special relativity in terms of kinematics
When was Emmy Noether born?
Emmy Noether was born on 23 March 1882 , the first of four children.
Who wrote the first computer virus?
$\Delta$
Whowrote the first computer virus?
Typing text
$\infty)^{2}$

Figure 2: A Demonstrator of our QA System. Answers can be given directly from Wikipedia documents applying the DocChat and Sentence Encoder technologies that we have implemented.

We have integrated this technology into our chatbot engine so that natural language questions can be answered directly from stored documents. In addition to the cluster-based navigation in text documents that we described above, we have the possibility to connect direct queries and possible answers from documents and add them to the user experience on the bot. This is especially useful when users have direct queries about compliance regulations, for example, or are looking for concrete instructions for action or process instructions.

\section{Summary}

The previous sections demonstrate where technological solutions help us to aim for reducing the configuration efforts in chatbot frameworks. In doing so, we addressed three aspects. First of all the connection of specialized applications can be done elegantly with RPA. Second, navigation within unstructured information can be done via a Conversational Interface by semantic clustering of text content without the need of manually transfering document content into the system. Thirdly, new technologies from the field of Open Domain Question Answering allow us to directly access information from texts by formulating natural language questions.

In the future, these options will make it easier to configure and integrate information into this communication channel, so that users in companies will not have to maintain complex duplicate structures and can attach the system directly to existing information sources. However, it is not only the technologies that are crucial here. In particular, the focus is on embedding the user experience during use and configuration. In our work, it has not yet been possible to decide whether full automation or ongoing support during configuration is superior. What is certain is that options 
for correcting errors made by ML components lead to higher acceptance. Here, the tradeoff between workload and service quality determines which paradigm will prevail. First experiments have been conducted and resulted in different ideas like wizards for importing and preparing documents to the system. This could be visual components for separating documents to meaningful units using a visual editor. For example, imported documents can be separated into paragraphs, sections or single sentences. This can be supported by visualizations of the documents and tools which allow users to slice the documents. The following investigations represent the next step for our work. We will now review in detail how these innovative methods of information integration can be meshed with an optimal user experience in this way. For this, we will primarily investigate the interaction with the components in the configuration GUI and Conversational Interface and learn together with pilot users. For this purpose, we have set up specific practical pilot applications in the areas of municipal applications, human resources and customer support.

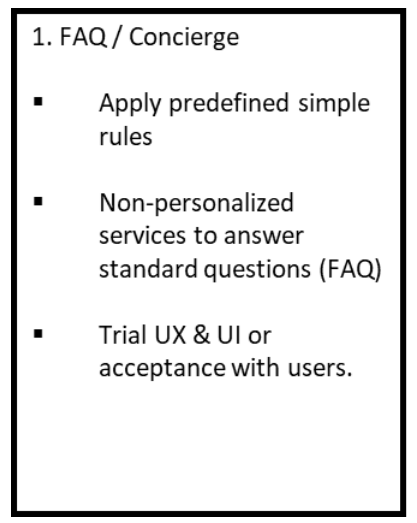

\begin{tabular}{|c|c|c|}
\hline $\begin{array}{l}\text { 2. Info service } \\
\text { - } \quad \text { Database queries } \\
\text { - } \quad \text { Form functions } \\
\text { - } \quad \text { Calculated answers } \\
\text { - } \quad \text { business applications } \\
\text { - Consultation dialogs }\end{array}$ & $\begin{array}{l}\begin{array}{l}\text { 3. Business application } \\
\text { (complete) }\end{array} \\
\text { - } \quad \text { Personalization } \\
\text { - } \quad \begin{array}{l}\text { Extensive integration } \\
\text { with databases and } \\
\text { business processes }\end{array} \\
\text { - Initiation \& monitoring } \\
\text { of business processes } \\
\text { - Connection of services } \\
\text { from ecosystems }\end{array}$ & $\begin{array}{l}\text { 4. Personal assistant } \\
\text { - } \quad \begin{array}{l}\text { Self-learning and - } \\
\text { adjusting }\end{array} \\
\text { - Automatic creation of } \\
\text { dialog patterns } \\
\text { - Derivation of Next Best } \\
\text { Actions } \\
\text { - Measuring success and } \\
\text { improving interaction }\end{array}$ \\
\hline
\end{tabular}

Figure 3: Illustration of a 4-phase maturity model for chatbots in which we have highlighted the state of the system and the maturity level classification of our developments in green. Note that despite the intelligent integration of external information via RPA and QA mechanisms, we are only at the 2nd stage and still have to go ahead to become a personal assistant.

The management and integration of external data is the first step to avoid the maintenance of redundant structures. In a 4-phase model of the maturity level of chatbots, the state of an info service can thus be achieved. Databases are integrated, forms can be filled out, answers can be calculated dynamically and we achieve a low-level integration of business applications. In the phase model in Figure 3, we show that the integration of information is a first step to provide voice assistants with advanced functionalities. We still have not reached a state where business applications and functionalities of a personal assistant are realized. Advanced functionalities, such as personalization, the integration of complete business processes or the connection of entire data ecosystems are further topics we have to take care of in the future. As a result, we are still some way off from the ultimate expansion stage to create a true personal assistant. The ability of independent learning, automatic dialog patterns or a prediction of possible actions are not yet available in many solutions but our work represents some promising steps towards this goal.

\section{Acknowledgement}

This work is funded by the SAB under project number 100395769. 


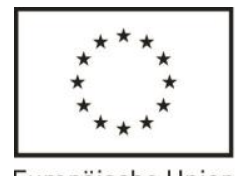

Europäische Union

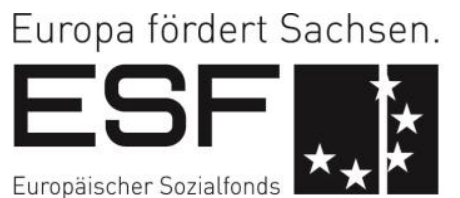

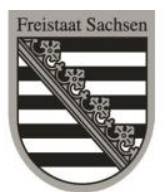

Diese Maßnahme wird mitfinanziert durch Steuermittel auf der Grundlage des vom Sächsischen Landtag beschlossenen Haushaltes.

\section{References}

Adam, M., Wessel, M., \& Benlian, A. (2020). AI-based chatbots in customer service and their effects on user compliance. Electronic Markets. https://doi.org/10.1007/s12525-02000414-7

Bojanowski, P., Grave, E., Joulin, A., \& Mikolov, T. (2017). Enriching Word Vectors with Subword Information. Transactions of the Association for Computational Linguistics, http://dx.doi.org/10.1162/tacl_a_00051

Brandtzaeg, P. B., \& Følstad, A. (2017). Why People Use Chatbots. In I. Kompatsiaris, J. Cave, A. Satsiou, G. Carle, A. Passani, E. Kontopoulos, S. Diplaris, \& D. McMillan (Hrsg.), Internet Science (Bd. 10673, S. 377-392). Springer International Publishing. https://doi.org/10.1007/978-3-319-70284-1_30

Cer, D., Yang, Y., Kong, S., Hua, N., Limtiaco, N., St. John, R., Constant, N., Guajardo-Cespedes, M., Yuan, S., Tar, C., Strope, B., \& Kurzweil, R. (2018). Universal Sentence Encoder for English. Proceedings of the 2018 Conference on Empirical Methods in Natural Language Processing: System Demonstrations, 169-174. https://doi.org/10.18653/v1/D18-2029

Dale, R. (2016). The return of the chatbots. Natural Language Engineering, 22(5), 811-817. https://doi.org/10.1017/S1351324916000243

Devlin, J., Chang, M.-W., Lee, K., \& Toutanova, K. (2019). BERT: Pre-training of Deep Bidirectional Transformers for Language Understanding. Proceedings of the 2019 Conference of the NACL: Human Language Technologies, http://dx.doi.org/10.18653/v1/N19-1423

Dunning, T. (1993). Accurate methods for the statistics of surprise and coincidence. Computational linguistics, 19(1), 61-74. https://dl.acm.org/doi/10.5555/972450.972454

Feine, J., Morana, S., \& Gnewuch, U. (2019). Measuring Service Encounter Satisfaction with Customer Service Chatbots using Sentiment Analysis. Wirtschaftsinformatik.

Ferrucci, D., Levas, A., Bagchi, S., Gondek, D., \& Mueller, E. T. (2013). Watson: Beyond jeopardy! Artificial Intelligence, 199, 93-105. https://doi.org/10.1016/j.artint.2012.06.009

Følstad, A., \& Skjuve, M. (2019). Chatbots for customer service: User experience and motivation. Proceedings of the 1st International Conference on Conversational User Interfaces - CUI '19, 1-9. https://doi.org/10.1145/3342775.3342784 
Jain, M., Kumar, P., Kota, R., \& Patel, S. N. (2018). Evaluating and Informing the Design of Chatbots. Proceedings of the 2018 Designing Interactive Systems Conference, 895-906. https://doi.org/10.1145/3196709.3196735

Mollá, D., \& Vicedo, J. L. (2007). Question answering in restricted domains: An overview. Computational Linguistics, 33(1), 41-61. https://doi.org/10.1162/coli.2007.33.1.41

S Shawar, B.A., \& Atwell, E. (2007). Chatbots: Are they Really Useful? LDV Forum, 22, 29-49.

Quasthoff U., Goldhahn D., Eckart T. (2014) Building Large Resources for Text Mining: The Leipzig Corpora Collection. In: Biemann C., Mehler A. (eds) Text Mining. Theory and Applications of Natural Language Processing. Springer, Cham. https://doi.org/10.1007/978-3-319-12655-5_1 r.

Voorhees, E. M. \& others. (1999). The TREC-8 question answering track report. Trec, 99, 77-82.

Yan, Z., Duan, N., Bao, J., Chen, P., Zhou, M., Li, Z., \& Zhou, J. (2016). DocChat: An Information Retrieval Approach for Chatbot Engines Using Unstructured Documents. Proceedings of the 54th Annual Meeting of the Association for Computational Linguistics (Volume 1: Long Papers), 516-525. https://doi.org/10.18653/v1/P16-1049 


\section{Author(s) Biography(ies)}

Andreas Niekler, Dr. Ing. Andreas Niekler is a research associate at the Institute of Computer Science at the University of Leipzig. He develops computer-based methods in the field of semantic properties in language and language-based AI. In recent contributions he supported various companies in Saxony adopting certain AI technologies in their daily activities. This includes processing of unstructured data for knowledge management and document management.

Mark Busse is the founder of the company $1000^{\circ}$ DIGITAL GmbH, which has been active on the market since 2000. With his team he develops innovative digital solutions for internal and external communication in industry contexts. With the ePaper $1000^{\circ}$ DIGITAL has had great success for many years. He is CEO and program manager of Kiko, the complete chatbot solution offered by $1000^{\circ}$ DIGITAL.

Felix Helfer, Msc. is a $\mathrm{PhD}$ candidate at Leipzig University. He is an expert in language generation and deep learning applications. In his recent research he investigated how natural sounding questions can be formulated from textural statements.

Matthias Gulde studies Computer Science at the Leipzig University. He is an expert in answer retrieval systems and a skilled Machine Learning engineer.

Lino Markfort MSc., is a Research Associate and PhD candidate at Leipzig University at the Chair of Innovation Management and Innovation Economics. His research focuses on digital business models, servitization and platforms. Most recently, he researched business model patterns and elements of IoT platforms. As an expert in the field of business model innovation, he supports $1000^{\circ}$ DIGITAL in strategy development. 